\title{
Characterization of HOPG, Sputtered HPOG and Graphene by ToF-SIMS and XPS
}

\author{
Chi-Ming Chan, Wenjing Xie, Lu-Tao Weng, Kai Mo Ng, Chak K. Chan \\ Hong Kong University of Science and Technology \\ Clear Water Bay, Kowloon, Hong Kong \\ kecmchan@ust.hk; wxiead@ust.hk; mcltweng@ust.hk; kmng@ust.hk; keckchan@ust.hk
}

\section{Extended Abstract}

Graphene, a single layer of graphite, has attracted much attention due to its physical properties and two-dimensional structure. It has been used in many important industrial applications, including batteries, electronic devices and sensors. The cleanliness of graphene surfaces is important for successful uses of graphene in these applications. Surfaces of highly oriented pyrolytic graphite (HOPG), which can be regarded as the surfaces of defect-free graphene and surfaces of graphene were characterized by two very powerful surface analysis techniques - X-ray photoelectron spectroscopy (XPS) and time-of-flight secondary ion mass spectrometry (ToF-SIMS) [1-4]. XPS results indicated that the impurities on the surfaces of graphene and HOPG can be removed by annealing samples of graphene and HOPG in vacuum at $400{ }^{\circ} \mathrm{C}$ [5].

In addition, ToF-SIMS results showed that even a small amount of poly(methyl methacrylate) (PMMA) impurity on the graphene surface can be removed by annealing the sample in vacuum at $500{ }^{\circ} \mathrm{C}$. In the $\mathrm{C} 1 \mathrm{~s}$ spectrum of clean $\mathrm{HOPG}$, an asymmetric $\mathrm{sp}^{2}$ carbon peak and a $\pi-\pi^{*}$ shake-up peak were present, indicating the absence of defects. An additional $\mathrm{sp}^{3}$ carbon peak representing sp3 defects was present, however, in the $\mathrm{C} 1 \mathrm{~s}$ spectrum of clean graphene. The positive ion spectra of these two materials differed somewhat, with the major difference being the presence of $\mathrm{C}_{\mathrm{x}} \mathrm{H}_{2}^{+\bullet}$ ions in graphene.

To determine the origin of the $\mathrm{C}_{\mathrm{x}} \mathrm{H}_{2}{ }^{+\bullet}$ ions, defects were created on the surface of HOPG by bombarding it with a $\mathrm{Cs}^{+}$ion beam at various sputtering doses. A sp ${ }^{3}$ carbon peak at $284.9 \mathrm{eV}$, representing $\mathrm{sp}^{3}$ defects appeared in the XPS spectra of sputtered HOPG samples, confirming that the surface of the sputtered HOPG is similar to that of graphene. A detailed examination of the positive ion spectra of ion-bombarded HOPG surfaces reveals the presence of the $\mathrm{C}_{\mathrm{x}} \mathrm{H}_{2}{ }^{+}$ions, confirming that these $\mathrm{C}_{\mathrm{x}} \mathrm{H}_{2}{ }^{+}$, which came from the defects created on the sputtered HOPG surfaces, are similar to the defects present on graphene surface.

\section{Acknowledgements}

The work described in this paper was fully supported by the Research Grants Council of the Hong Kong Special Administrative Region, China (grant nos. 600513 and 16300314)

\section{References}

[1] C. M. Chan, Surface Modification and Characterization of Polymers. Hanser Press, 1994.

[2] C. M. Chan, L. T. Weng, and Y-T. R. Lau, "Polymer Surface Structures Determined Using ToF-SIMS," Reviews in Analytical Chemistry, vol. 33, pp. 11-30, 2014.

[3] L. T. Weng and C. M. Chan, "Surface Analysis," in Wiley Encyclopedia of Composites, L. Nicolais, A. Borzacchiello, and S, M. Lee, Ed., Wiley, 2012.

[4] L. T. Weng and C. M. Chan, "Characterization of Polymeric Materials," in ToF-SIMS - Surface Analysis by Mass Spectrometry, J. Vickerman, $2^{\text {nd }}$ Ed., SurfaceSpectra Ltd/IM Publications LLP, pp. 503-530, 2013.

[5] W. Xie, L-T. Weng, K. M. Ng, C. K. Chan, and C-M. Chan, "Clean Graphene Surface through High Temperature Annealing," Carbon, vol. 94, pp. 740-748, 2015. 